
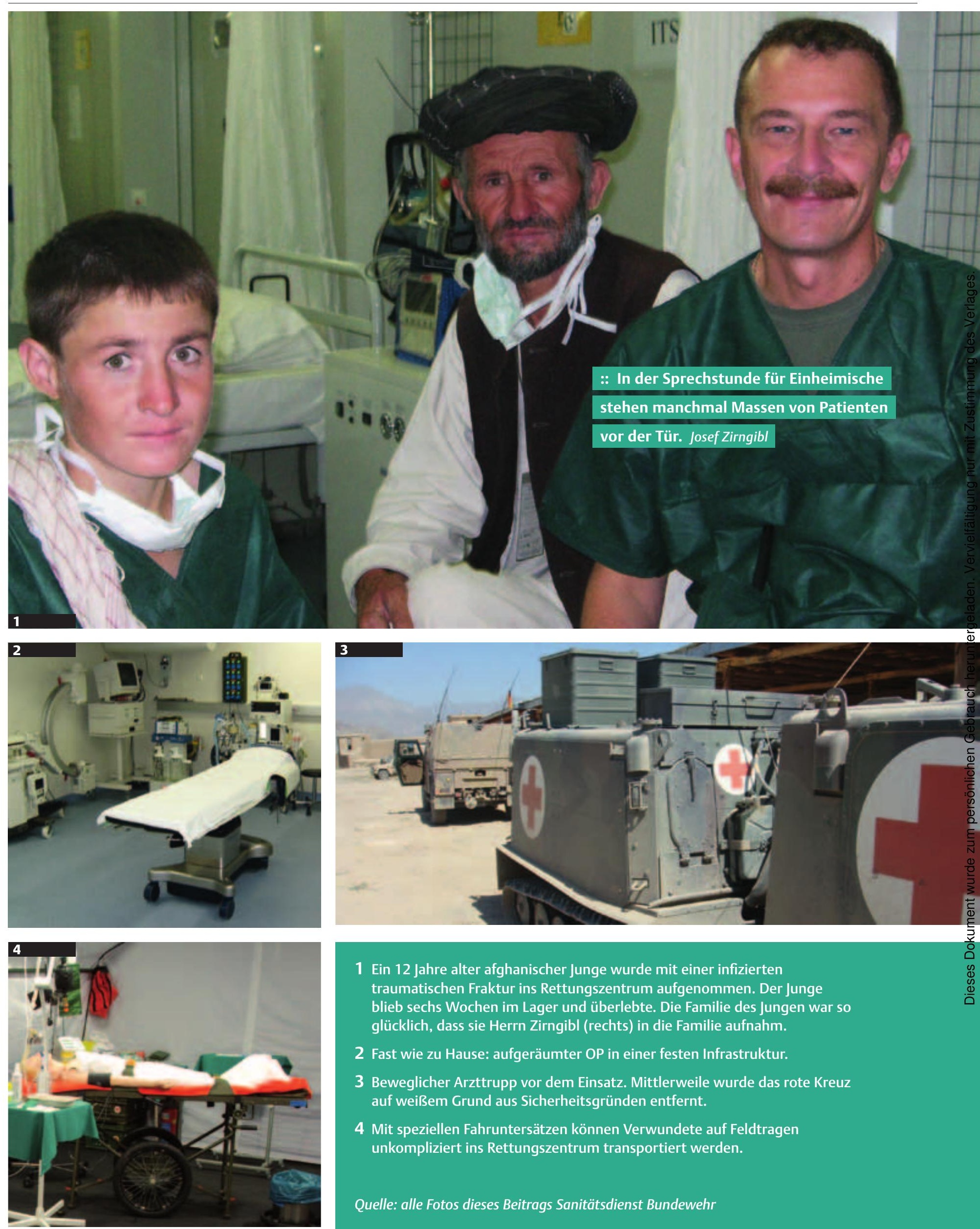

1 Ein 12 Jahre alter afghanischer Junge wurde mit einer infizierten blieb sechs Wochen im Lager und überlebte. Die Familie des Jungen war so glücklich, dass sie Herrn Zirngibl (rechts) in die Familie aufnahm.

2 Fast wie zu Hause: aufgeräumter OP in einer festen Infrastruktur.

3 Beweglicher Arzttrupp vor dem Einsatz. Mittlerweile wurde das rote Kreuz auf weißem Grund aus Sicherheitsgründen entfernt.

4 Mit speziellen Fahruntersätzen können Verwundete auf Feldtragen unkompliziert ins Rettungszentrum transportiert werden. 


\section{Ohne Netz und doppelten Boden}

ALS OTA IN AFGHANISTAN „Zu Hause im OP einer schönen Klinik kenne ich mich aus. Ich weiß, wo ich was finde, und zur Not frage ich nach. Im Auslandseinsatz dagegen muss ich mich von der gesamten technischen Ausrüstung in einem OP bis zur medizinischen Versorgung des Patienten perfekt auskennen. Ich muss darauf vorbereitet sein, dass jede Sekunde etwas passieren kann, und keiner hinter mir steht, den ich fragen kann. Im Auslandseinsatz ist man in einem kleinen Team auf sich allein gestellt.“

So beschreibt Oberstabsfeldwebel Josef Zirngibl eine der großen Herausforderungen bei seiner Arbeit in einem Krisengebiet. Zirngibl ist OP-Fachpfleger und seit seinem ersten Einsatz in Kroatien vor 14 Jahren regelmäßig für die deutsche Bundeswehr im Ausland tätig. Zuletzt war Zirngibl für drei Monate in Feyzabad in Afghanistan eingesetzt. In dieser Zeit führte er in seinem Team 134 Operationen durch. Zirngibl war bereit, uns ausführlich von seiner Arbeit im Krisengebiet zu berichten.

\section{Operationssäle aus dem Baukasten}

Ein OP der Bundeswehr im Einsatz kann in einem Zelt, einem Container oder einem festen Gebäude beherbergt sein. Ein Zelt wird verwendet, wenn Auf- und Abbau sowie der Einsatz selbst schnell laufen müssen. Wird dagegen angenommen, dass der Einsatz bis zu sechs Monate dauern wird, benutzt man Container, die durchaus auch einige Jahre verwendet werden können. Sobald man davon ausgeht, dass es sich um einen längeren Einsatz handelt, wird versucht, feste Gebäude und Infrastrukturen zu errichten - wie sie im Moment im Kosovo und in Afghanistan bestehen.

Alle Materialien und Geräte, vom Instrumentarium bis zur Klimaanlage, und die äußere Hülle des OPs werden in Deutschland auf Paletten platzsparend verpackt und in das entsprechende Gebiet transportiert. Theoretisch können jederzeit und überall auf der Welt Zelte oder Container mit unterschiedlichen Funktionen nach dem „Baukastenprinzip“ aufgestellt werden. Ein Team, welches das entsprechende Gebiet vorher begutachtet, bestellt in Deutschland beispielsweise einen OP-Container mit Geräteversorgungs-Container und Operationsvorbereitungs-Container. Hinzu kommt ein Zugangs-Container, der alle drei, sozusagen als Eingang, verbindet (Abb. Rettungszentrum).

Je nach Bedarf können aus dem „Baukasten“ auch eine Intensivstation, eine Pflegestation, eine Apotheke oder weitere OPs bestellt und jederzeit hinzugefügt werden. Die Paletten werden dann von Deutschland aus in die nächstgelegene Stadt des Einsatzgebietes geflogen und von dort meist mit Lkws weiter transportiert. Eine speziell ausgebildete Truppe baut alles auf, sodass die OP-Gruppe in kürzester Zeit ihre Arbeit aufnehmen kann.

\section{Ziel: „Nur“ Erstversorgung}

Jeder OP-Saal wird von einer OP-Gruppe betrieben, die aus sechs bis sieben Personen besteht, darunter ein bis zwei OP-Fachpfleger oder Operationstechnische Assistenten und ein Anästhesie-
Fachpfleger. Von ärztlicher Seite gehören ein Arzt, der meist die Facharztbezeichnung Chirurgie trägt, ein Assistenzarzt und ein Anästhesist mit einem Weiterbildungsassistenten für das Fach Anästhesie zum Team. Am schwierigsten ist die Arbeit im Zelt. Es besteht aus einem festen Boden und einer aufblasbaren Hülle, die durch spezielle Kammern stabilisiert wird. Feste Wände und Decken gibt es nicht, was vor allem die Einrichtung, wie beispielsweise das Aufhängen von Schläuchen oder die Abtrennung von unreinem und reinem Bereich, erschwert. Vor Ort baut die zuständige Einheit innerhalb von zwölf Stunden die Zeltkomponente auf, räumt sie ein, und führt eine Schlussdesinfektion durch. Eine weitere Stunde braucht die OP-Gruppe, um die Vorbereitungen für den ersten Eingriff zu treffen. In dem etwa 20 Quadratmeter großen Zelt besteht die Aufgabe des OP-Teams darin, das Leben der durch Kampf oder Anschläge verletzten Kameraden zu retten. Es geht also in erster Linie um die notfallmedizinische Erstversorgung. Das können chirurgische Eingriffe mit postoperativer Überwachung, aber auch die Versorgung von Verletzungen sein.

Ziel ist es, dass der schwer verletzte Soldat nach der Erstversorgung in ein Krankenhaus vor Ort oder nach Deutschland transportiert werden kann. „Wenn es um Leben oder Tod geht, kann

\section{RETTUNGSZENTRUM DER BUNDESWEHR}

GVC = Geräte-Versorgungscontainer: versorgt die OP-Gruppe mit Trinkwasser, Druckluft und Sauerstoff. OPV = Operationsvorbereitungs-Container: dient auch zur Lagerung von Sanitätsmaterialien. Z = Zugang: kann fünf Funktionscontainer miteinander verbinden.

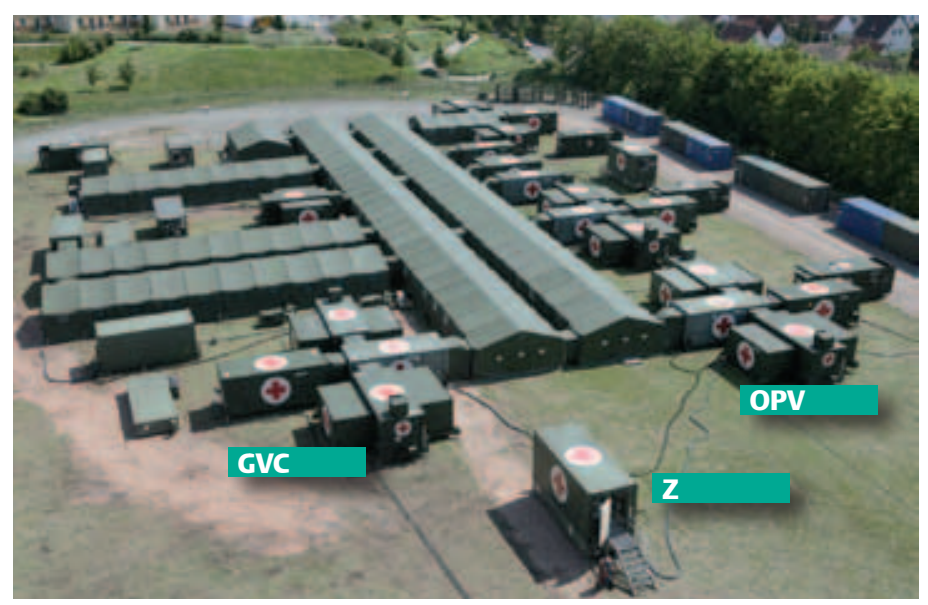


ich mich nicht mit Oberschenkelbrüchen aufhalten, sondern muss schauen, dass der Verletzte überlebt. In solchen Fällen wird der Patient mit Fixateur externe und Analgetika ruhig und schmerzfrei gestellt, damit er in eine Einrichtung verlegt werden kann, in der er angemessen weiter behandelt wird. Haben wir die Zeit und die Möglichkeit, wird der Patient auch vor Ort weitergehend versorgt", schildert Zirngibl. Er sieht den limitierenden Faktor oft in den Fähigkeiten des medizinischen Personals: „Wir brauchen bei der Bundeswehr die berühmte eierlegende Wollmilchsau, das heißt, der Operateur und das Pflegepersonal müssen vom Kopf bis zur großen Zehe alles abdecken." Das geht gegen den Trend in der Medizin, der Pflegepersonal und Ärzten immer stärkere Spezialisierung abverlangt. Dadurch wird auch die Suche der Bundeswehr nach Allroundern schwieriger.

\section{Hochwertige Materialien in einem ausgeklügelten System}

Aus technischer Sicht dagegen sind dem OP-Team kaum Grenzen gesetzt. Zur Diagnosestellung und intraoperativen Unterstützung stehen auch einem kleinen Rettungszentrum wie in Kunduz oder Feyzabad ein digitales mobiles Röntgengerät, ein kleines tragbares Ultraschallgerät und ein Mini-Labor zu Verfügung. Das Labor wird von einem MTA betrieben und kann die wichtigsten Werte, zum Beispiel Blutgase, ermitteln. Ergänzend ist sogar eine kleine Blutbank mit circa 35 Erykonzentraten vorhanden.

Größere Einrichtungen wie in Masar-e Sharif entsprechen in etwa dem Standard eines Kreiskrankenhauses. Hier steht so gut wie alles bis hin zum CT zur Verfügung.

Aber auch die Ausstattung im OP eines Containers beziehungsweise Zeltes (Kasten rechts) kann sich sehen lassen. Sie unterscheidet sich im Grundsatz nicht von der Ausstattung eines deutschen OP-Saals. Mit einer Klimasteuerung kann die OP-Gruppe die gewünschte Temperatur im OP einstellen, den Strom für den Betrieb spendet ein Generator. Fällt die primäre Stromversorgung aus, schaltet sich eine akkubetriebene Notversorgung ein, die die OP-Lampe und das Narkosegerät versorgt. Die Außenluft, die durch die Anlage gekühlt in den OP gelangt, durchläuft vorher einen Filter, der im Normalfall alle zwei Wochen ausgewechselt

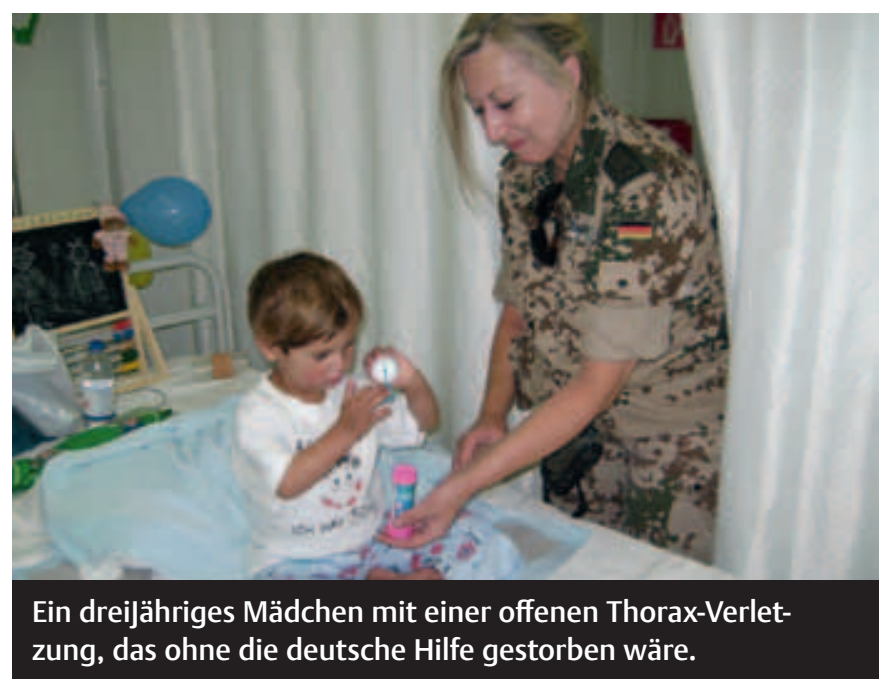

werden muss. Wenn es Sandstürme gibt, wie es in Afghanistan hin und wieder der Fall ist, müssen die Filter täglich ausgetauscht werden.

Die Bundeswehr beschäftigt ganze Stäbe, die austüfteln, wie der "tragbare OP“ noch kleiner und leichter werden kann, ohne dass die Qualität darunter leidet. Zum Beispiel wurde ein neues Notfallinstrumentarium entwickelt, das auf ein Minimum reduziert ist, um Transportgewicht zu sparen. Darin sind 15 Grundsiebe und sieben Ersatzsiebe enthalten. Die Neuheit nennt sich rDCS (ressourcenorientierte Damage-Control-Surgery) und soll bei Einsätzen verwendet werden, in denen es besonders schnell gehen muss und die Transportkapazität eingeschränkt ist - zum Beispiel wenn eine OP-Gruppe 15 Verletzten innerhalb von 24 Stunden eine adäquate Erstversorgung bieten muss. Der Umfang eines Grundsiebs für den Einsatz ist größer als ein Basissieb in einem

\section{Das Instrumentarium und die Geräte im Einsatz}

sind sehr hochwertig und erlauben jegliche Versor-

gung, aber die Handhabung ist ein bisschen anders

als bei den gewohnten Geräten in Deutschland.

„normalen“ OP. Zudem kann der Inhalt des Siebs an die erwarteten Verletzungen angepasst werden, so wie auch im Zivilen ein Basissieb in der Viszeralchirurgie anders aussieht als in der Orthopädie.

„Das Instrumentarium und die Geräte im Einsatz sind sehr hochwertig und erlauben jegliche Versorgung, aber die Handhabung ist ein bisschen anders als bei den gewohnten Geräten in Deutschland. Im Einsatz werden andere HochfrequenzchirurgieGeräte für die Koagulation verwendet als in deutschen Krankenhäusern. Auch der Fixateur externe nach Stuhl-Heiser wurde in Deutschland schon durch modernere Methoden der Fixation abgelöst“, erläutert Zirngibl. Er sieht aber die Problematik nicht in der Bedienung der Geräte, sondern in der Ausbildung des Personales bzw. der standardisierten Arbeitsweise des Personals: „Wenn ich einen Raum betrete und weiß, der Lichtschalter ist auf der rechten Seite, habe ich kein Problem. Ist er aber plötzlich links, tappe ich im Dunkeln. Deshalb ist es erforderlich, dass man die-

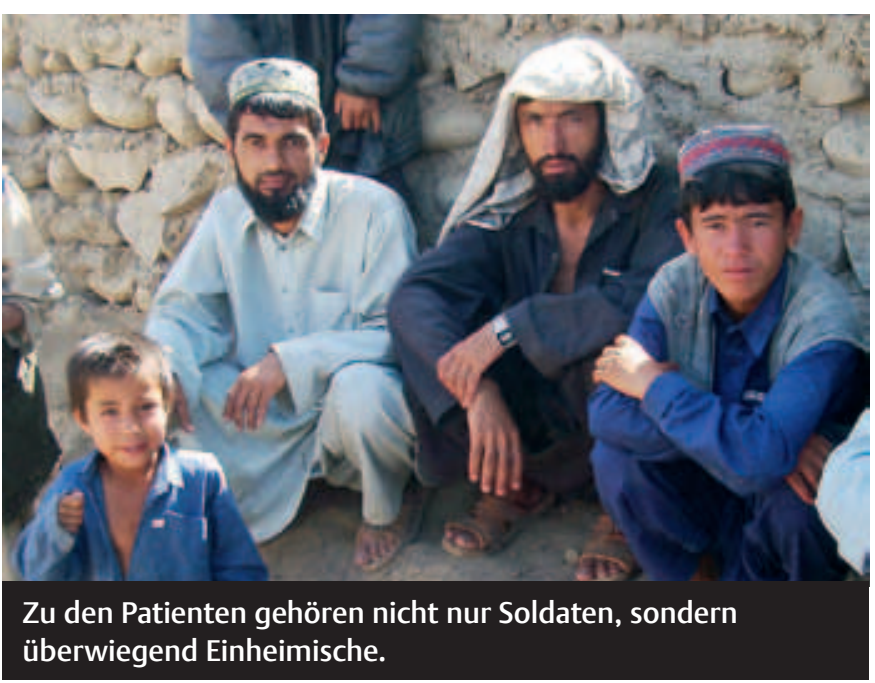


se Unterschiede der Handhabung vor dem Einsatz erlernt. Dann kommt jeder Neuling sehr gut zurecht. Derzeit erklärt das erfahrene Personal diese Dinge den neuen Einsatzkräften vor Ort.“

\section{Moderner Schutz und Terrorismus verändern Verletzungsmuster}

Zwar kann in einem OP-Zelt in Afghanistan theoretisch jede Operation durchgeführt werden. In der Realität kommen aber meist ähnliche Verletzungsmuster vor. Die meisten Soldaten werden heute im Gesicht, besonders an Mund und Kiefer, oder an den Extremitäten verletzt, denn Thorax und Bauchraum sind durch schwere schusssichere Westen (Bristols) sehr gut geschützt. „In letzter Zeit haben wir häufig Schussverletzungen durch den direkten Beschuss mit Handfeuerwaffen, wie beispielsweise mit einer Kalaschnikow. Wenn es glimpflich ausgeht, leidet der Soldat unter einem Knalltrauma, einem HWS-Syndrom, Splitterverletzungen und manchmal auch an einer Durchschussverletzung an den Extremitäten“, berichtet Zirngibl aus seiner Erfahrung. Hinzu kommen in den Zeiten terroristischer Anschläge die „blast injuries“ - Verletzungen, die durch Explosionen und die dadurch verursachten Druckwellen entstehen. Dazu zählen sowohl Lungenschäden wie Ödeme, die die Druckwelle selbst auslöst, oder Verätzungen, wenn giftige Gase eingeatmet werden. Aber auch Verletzungen durch Splitter und Gegenstände, die bei einer Detonation durch die Luft schießen, kommen vor.

Gerade bei Explosionen kann es passieren, wie es in Kunduz schon der Fall war, dass fünf Schwerverletzte auf einmal versorgt werden müssen. „In Deutschland wäre eine Klinik mit drei polytraumatisierten Patienten überfordert. Im Einsatzgebiet muss das eine einzige OP-Gruppe meistern. Dabei muss man vor der Behandlung erst einmal eine Art Ranking durchführen: Wer braucht am nötigsten Hilfe? Wer hat die größten Überlebenschancen? Wer kann warten? Und in ganz seltenen Fällen muss man leider vorher zugeben, dass es hoffnungslos ist." Durchschnittlich operiert eine OP-Gruppe ein bis zwei Patienten am Tag, wobei Notfälle nicht mit eingerechnet sind.

\section{: Wenn wir den Kindern helfen, ist die Wahrschein-}

lichkeit geringer, dass deren Eltern oder Verwandte die

\section{Taliban unterstützen.}

\section{Behandlung Einheimischer verbessert das Ansehen der Truppe}

$\mathrm{Zu}$ den Patienten gehören aber nicht nur Soldaten, sondern überwiegend Einheimische. Diese Patienten kommen auf verschiedenen Wegen zu den Deutschen, zum Beispiel über eine kostenfreie Sprechstunde, die - wenn es die Lage zulässt - jeden Nachmittag stattfindet. So kann die OP-Gruppe humanitäre Hilfe leisten, mit den dortigen Bewohnern das wichtige Vertrauensverhältnis aufbauen und ihre eigenen medizinischen Fähigkeiten durch praktische Übung erhalten. Zirngibl hat auch schon erlebt, dass erkrankte Einheimische einfach vor dem Tor abgelegt werden. Es gibt aber auch Fälle, in denen inländische Ärzte die Deutschen zur

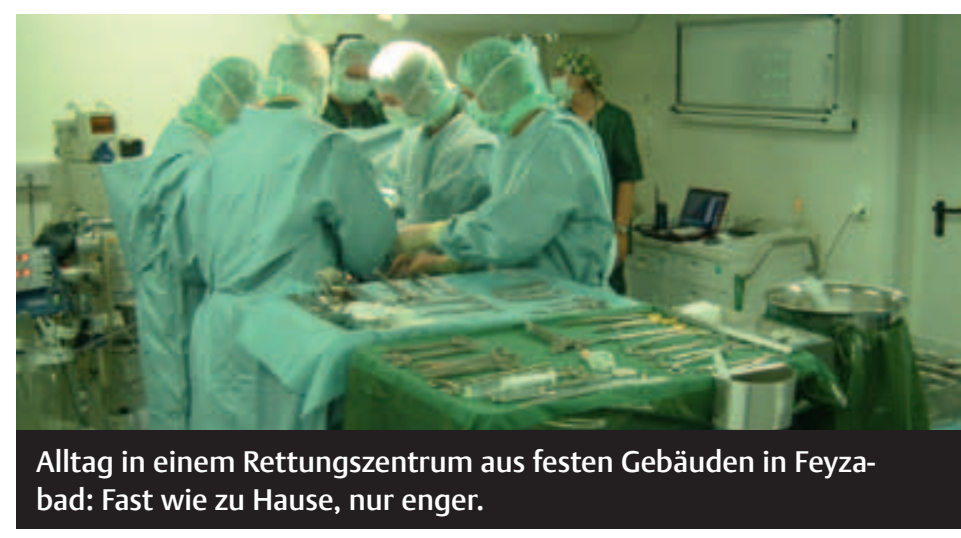

\section{AUSSTATTUNG IN EINEM OP-ZELT ODER -CONTAINER}

\begin{tabular}{|c|c|}
\hline Relaxometer & OP-Leuchte \\
\hline Patientenüberwachungsmonitor & Auftau-/Wärmegerät \\
\hline Elektrokardiograf & Beatmungsgerät \\
\hline Defibrillator & Ständer, Schale \\
\hline Infusionspumpe & Intubationsbesteck \\
\hline Injektionspumpe & Chirurg. Einsatz-Instrumentarium \\
\hline Künstliche Beatmung & Narkosegerät \\
\hline Röntgenbetrachtungsgerät & Gasversorgungsanlage \\
\hline Untersuchungsleuchte & OP-Sauggerät \\
\hline Infusionsständer & Gasversorgungsanlage \\
\hline Narkosewagen & Hochfrequenzchirurgie-Gerät \\
\hline Infusionsständer & Hochfrequenzchirurgie-Gerät \\
\hline Narkosewagen & Klimasteuerung \\
\hline OP-Tisch & OP-Eimer \\
\hline OP-Hocker & Instrumenten-Zureichtisch \\
\hline Blutkonservenkühlschrank & Infusionsgerät \\
\hline Wäsche- und Abfallsammler & \\
\hline \multicolumn{2}{|c|}{$\begin{array}{l}\text { Die Ausstattung ist variabel und kann an den Einsatz angepasst } \\
\text { werden. Wenn bestimmte Verletzungen häufiger erwartet werden, } \\
\text { kann das Instrumentarium spezifisch aufgestockt werden. Ist die } \\
\text { Transportkapazität an einem Einsatzort dagegen stark eingeschränkt, } \\
\text { kann die Ausstattung reduziert werden. }\end{array}$} \\
\hline
\end{tabular}

Hilfe holen, um sich bei einem schwierigen Patienten beispielsweise eine zweite Meinung einzuholen. „In der Sprechstunde für die Einheimischen stehen manchmal Massen von Patienten vor der Tür. 15 bis 20 Patienten werden dann vorgesichtet hereingeholt. Denn wir nehmen nur die Fälle an, die wir auch behandeln können. Wenn jemand an einem Nierentumor erkrankt ist und wir operieren ihn, dann würde er dialysepflichtig werden. Ist die nächste Dialyse aber zu weit weg, können wir - so leid es uns tut so einen Fall nicht behandeln“, bedauert Zirngibl. Besser sieht es dagegen für Patienten aus, denen eine Extremität amputiert werden muss, denn in Feyzabad und Masar-e Sharif hat eine zivile Hilfsorganisation je eine kleine Prothesen-Werkstatt eingerichtet. „Wenn ein Patient einen Ulcus am Bein hat, können wir es abnehmen, bevor es zur generalisierten Sepsis kommt. In so einem Fall ist die Amputation gut planbar und wir vermitteln frühzeitig eine Anschlussbehandlung, damit der Patient eine gute Prothese bekommt“, berichtet Zirngibl.

Es kommen aber auch akute Amputationen, beispielsweise bei Minenopfern, vor. Zirngibl erinnert sich an einen Einsatz, wo in 


\section{ZAHLEN}

Im Schnitt sind zwischen $\mathbf{4 3 0 0}$ und $\mathbf{4 4 0 0}$ Soldaten in Afghanistan im Einsatz, davon sind ca. 190 im Sanitätsdienst tätig, darunter acht OTAs/OP-Fachpfleger. In Masar-e Sharif gibt es zwei OP-Gruppen mit zusammen vier OTAs/OP-Fachpflegern. In Feyzabad und Kunduz arbeitet je eine OP-Gruppe à zwei OTAs/OP-Fachpfleger.

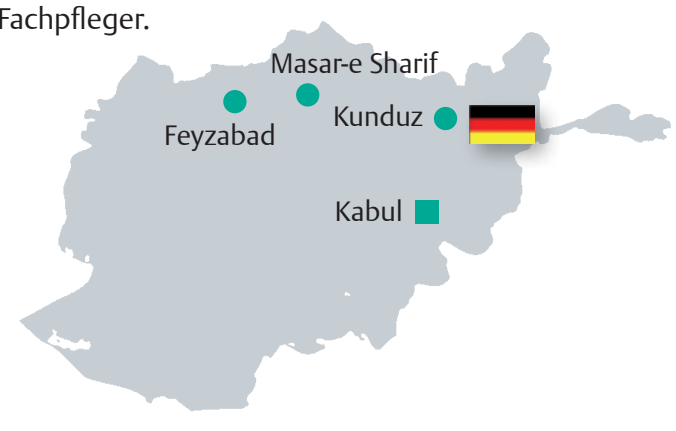

der Nähe des Sanitätsstützpunktes Feyzabad eine neue Straße in das ungefähr 150 Kilometer entfernte Taloqan gebaut wurde. Einheimische Bauarbeiter hoben das Gebiet aus, ohne es vorher von Minen zu befreien. So kamen in kürzester Zeit vier junge Männer in das deutsche Lager, deren Minenverletzungen versorgt werden mussten.

Bei Zirngibls letztem Einsatz mussten drei Kinder behandelt werden, die ohne die Hilfe des OP-Teams gestorben wären. Gerade bei der Rettung von einheimischen Kindern beschreibt Zirngibl zum einen das gute Gefühl des „Lebenrettens“, aber auch das verbesserte Ansehen der deutschen Truppen in der Bevölkerung: „Wenn wir den Kindern helfen, ist die Wahrscheinlichkeit geringer, dass deren Eltern oder Verwandte die Taliban unterstützen oder sonstige kriminelle Energie gegen die Bundeswehr einsetzten."

\section{Ein ganz normaler Arbeitstag - nur anders}

So hat es ein OP-Team mit den unterschiedlichsten Krankheitsbildern und Schicksalen zu tun. Um das zu bewältigen, sind die Aufgaben zwar wie überall klar verteilt. Wenn aber Not am Mann ist, springt jeder für alles ein, zum Beispiel beim Sterilisieren der Instrumente. Dafür gibt es eine fest installierte Sterilisationseinrichtung, die von ein bis zwei Sterilgutassistenten bedient wird. Wenn hier Hilfe gebraucht wird, hilft ein OTA, Pfleger oder Arzt, das Instrumentarium zu sterilisieren.

Verläuft alles normal, kümmert sich jeder um seinen eigenen Bereich. So sind die OTAs oder OP-Fachpfleger für die Vorbereitung des OPs zuständig. Nach dem Frühstück in der Kantine bespricht die OP-Gruppe den OP-Plan-für den kommenden Tag und die OTAs oder OP-Fachpfleger bereiten dementsprechend Instrumente, Verbandmaterialien und die Gerätschaft vor. Dann geht es in den Routinebetrieb über, mit dem Unterschied, dass zu jeder Zeit schwer verletzte Kameraden eintreffen können oder sogar das Lager selbst beschossen werden kann, wie es in Afghanistan vor allem in Kunduz bereits mehrfach vorkam. Die OP-Gruppe versucht, gegen 13 Uhr mit dem Tagesgeschäft fertig zu sein, da- mit der OP aufbereitet und die Instrumente zum Sterilisieren gegeben werden können und der OP für Notfälle bereitsteht.

Nach dem Mittagessen steht dann in der Notaufnahme die Sprechstunde für die Einheimischen auf dem Programm, bei der die OTAs/OP-Fachpfleger mit eingesetzt werden. Gegen 16:30 Uhr werden die Sprechzimmer geputzt und desinfiziert, anschließend bespricht die OP-Gruppe die Vorkommnisse des Tages mit dem Chef der Sanitätskompanie und kann in die wohlverdiente Dienstunterbrechung gehen. „Feierabend“ gibt es nicht, denn im Notfall müssen alle zur Verfügung stehen. In jedem Lager gibt es Betreuungseinrichtungen, die die Soldaten und das OP-Team am Abend besuchen können. Im Rahmen der „Zwei-Dosen-Regelung“ darf man hier Bier trinken oder antialkoholische Getränke zu sich nehmen. Manche spielen Billard oder sitzen zusammen und plaudern, andere machen in einem Sport-Container Fitnessübungen oder leihen sich in der Bibliothek Bücher aus. Zirngibl sitzt im Sommer am liebsten mit Kameraden vor den Arbeitsbereichen und unterhält sich mit ihnen.

\section{OTAs und OP-Fachpfleger sind rar und gern gesehen}

Die Bundeswehr hat Bedarf an gut ausgebildete OTAs sowie OPFachpflegern. Alle Bewerber sind sehr willkommen und können sich bei jeder Dienststelle bewerben. Anwärter müssen vor Dienstantritt eine dreimonatige Grundausbildung absolvieren, die eventuell verkürzt werden kann. Denn auch im Sanitätsdienst muss man sich insbesondere im Auslandseinsatz militärisch richtig verhalten können. Innerhalb einer sechsmonatigen Eignungsübung, die der Probezeit an zivilen Arbeitsplätzen entspricht, können beide Parteien entscheiden, ob sie ein gemeinsames Arbeitsverhältnis eingehen wollen.

Zirngibl begrüßt vor allem erfahrenes Personal, das schon einiges gesehen hat: „Es gibt im Ausland immer wieder Situationen und Begebenheiten, die man zuhause nicht erlebt. Deshalb ist es sehr wichtig, dass jeder im Team sein Wissen und seine Erfahrung, auch außerhalb seines Arbeitsbereiches, einbringt.

Wenn ein OTA oder OP-Fachpfleger in einem zivilen Krankenhaus angestellt bleiben und trotzdem an einem Auslandseinsatz teilnehmen möchte, gibt es die Möglichkeit - sofern der Arbeitgeber einverstanden ist - ihn für eine bestimmte Zeit freizustellen. Normalerweise dauert ein Einsatz für OTAs oder OP-Fachpfleger zwei Monate, während die anderen Soldaten sich meist vier Monate im Auslandseinsatz befinden.

\section{Mit der Patrouille das Lager verlassen}

Im Auslandseinsatz gibt es eine Vielzahl von Tätigkeitsbereichen für medizinisches Assistenzpersonal, unter anderem im Beweglichen Arzttrupp (BAT, geschützte militärische Rettungswagen Foto 3, Seite 4). Der BAT ist Teil einer Patrouille, damit im Ernstfall verletzte Soldaten vor Ort versorgt werden können oder zur weiteren Behandlung in das Lager gebracht werden können. Das Team der Notfall-Aufnahme holt den Verwundeten dann mit einem „Feldtragefahrgerät“ (Foto 4, Seite 4) aus dem militärischen Rettungswagen ab und fährt ihn, wenn nötig, direkt in den OP. Diese besondere Liege hat zwei große Reifen, damit Erschütterungen besser ausgeglichen und ungleichmäßiger Boden leichter zu 
bewältigen ist. Sie kann von einer einzelnen Person ohne Hilfsmittel aufgestellt werden.

Jede Truppe, die das Lager verlässt, wird von einem BAT begleitet und hat großes Vertrauen in das Sanitätsteam, das bei jeder Verwundung sofort zur Stelle ist. Im BAT mit auf Patrouille zu gehen ist gefährlich. Die Gefahr, beschossen zu werden, ist draußen natürlich größer als im Lager. „Vor kurzem wurde in Afghanistan ein Sanitätsfahrzeug beschossen. Dabei ist ein Oberstabsarzt gefallen“, beklagt Oberstleutnant Peter Wilhelm Fuss, der die Informationsarbeit im Sanitätsamt der Bundeswehr leitet. „Im Kriegsvölkerrecht steht, dass man keine Sanitätsfahrzeuge beschießen darf. Die Taliban suchen sich aber gezielt gerade Rettungsfahrzeuge aus“, erläutert er. Deshalb wurde das Symbol der Sanitätsfahrzeuge - das rote Kreuz auf weißem Grund - vom Schutzzeichen zum Gefährdungszeichen und musste von den militärischen Rettungswägen abgenommen werden. „Wenn die Patrouille heute fährt, kann man von außen nicht erkennen, wo sich der BAT befindet“, erklärt Fuss, der selbst als Heeresoffizier im Kriegsgebiet tätig war.

\section{Psychische Belastung - großer Zusammenhalt}

Ein Auslandseinsatz in Krisengebieten stellt immer eine psychische Belastung dar. In einem fremden Land, von Freunden und Familie getrennt, lebt man als Soldat für eine bestimmte Zeit auf engstem Raum mit den Kameraden. Täglich kann eine böse Überraschung ihr Leben gefährden. Im schlimmsten Fall kehrt der Soldat, Arzt oder Pfleger nicht nach Hause zurück oder muss den Tod eines Kameraden verkraften. Diese psychische Dauerbelastung

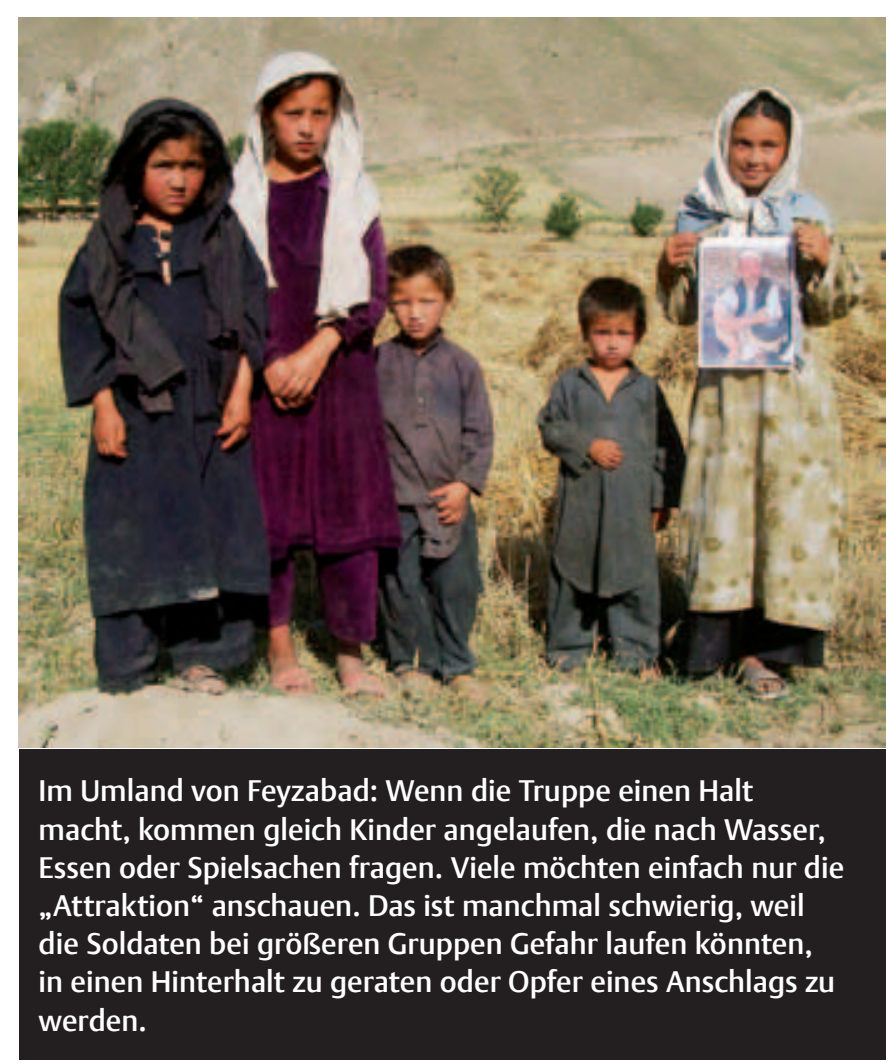

wurde lange Zeit, zum Teil auch heute noch, nicht thematisiert. Sicherlich wird diese Belastung individuell unterschiedlich aufgenommen und verarbeitet, aber sie besteht für jeden Einzelnen im Auslandseinsatz, und kann zu einer posttraumatischen Belastungsstörung führen. Vor allem Soldaten, die selbst Opfer von Gewalttaten werden oder mit der Verwundung oder dem Tod eines Kameraden konfrontiert werden, sind gefährdet, eine posttraumatische Belastungsstörung zu entwickeln. Die betroffenen Soldaten werden u.a. in vier Bundeswehrkrankenhäusern umfassend behandelt. Kürzlich hat in Berlin das Traumazentrum eröffnet, indem die Ergebnisse aus der Forschung umgehend in Praxis einfließen sollen, aber auch Erkenntnisse aus der Praxis durch die Forschung evaluiert werden.

Für alle aus dem Auslandseinsatz Zurückgekehrten gibt es Nachbereitungsseminare.. Zirngibl moderiert solche Seminare: „Dabei haben die Soldaten die Möglichkeit im Kameradenkreis den Einsatz gedanklich Revue passieren zu lassen und über Erlebtes zu sprechen. Wenn es gewünscht wird, kann professionelle Hilfe in Anspruch genommen werden, um das Geschehene zu verarbeiten." Viele Dinge, die während des Einsatzes vorfallen, werden noch dort von der Gruppe aufgefangen. Meist bestehen die Gruppen aus eingespielten Teams, in denen sich jeder völlig auf den anderen verlassen kann. Das schweißt zusammen, sodass hier meist auch besprochen wird, was den Einzelnen belastet. Dieser Zusammenhalt im Team wird von vielen Auslandskräften als überaus positiv und aufbauend beschrieben. Soldaten die sich nicht trauen, innerhalb der Gruppe Schwäche zu zeigen, haben die Möglichkeit, sich über eine 24-Stunden-Telefonhotline oder über ein anonymes Emailpostfach professionelle Hilfe zu holen. Mittlerweile nutzen auch Angehörige diese Angebote, wenn sie „Veränderungen" feststellen.

In Deutschland arbeitet Zirngibl mittlerweile im Sanitätsamt. Der Oberstabsfeldwebel kümmert sich um die OP-Ausstattung der Bundeswehrkrankenhäuser, aber auch die der Auslandseinsätze. Obwohl er nicht mehr verpflichtet ist, selbst an Auslandseinsätzen teilzunehmen, nimmt er jedes Jahr freiwillig erneut die Gefahren auf sich. Der OP-Fachpfleger wünscht sich, dass seine Kameraden fachgerecht versorgt werden: „Es gibt nicht so viele Leute mit langjähriger OP-Erfahrung in Krisengebieten. Ich mache das jetzt schon seit 24 Jahren und habe in dieser Zeit unendlich viel gelernt. Davon sollen meine Kameraden profitieren, schließlich legen sie ihr Leben in unsere Hände.“

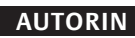

Mona Herz

Journalistin und Medizinstudentin

Freie Autorin des Georg Thieme Verlags seit 2008

\section{BIBLIOGRAFIE}

DOI 10.1055/s-0030-1268020

ImOP 2011; 1: 4-9

(c) Georg Thieme Verlag KG

Stuttgart . New York . ISSN 1611-7905 